\title{
DISTINGUISHING INTRACEREBRAL HEMORRHAGE FROM ACUTE CEREBRAL INFARCTION THROUGH METABOLOMICS
}

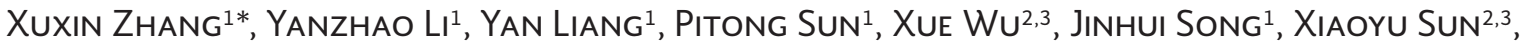 \\ Mo HONG ${ }^{2,3}$, PENG GAO ${ }^{2,4,5}$ AND DONGFENG Deng ${ }^{1 *}$ \\ ${ }^{1}$ Department of Neurosurgery, Affiliated Zhongshan Hospital of Dalian University, Dalian, Liaoning; ${ }^{2}$ Key Laborotary of \\ Liaoning Tumor Clinical Metabolomics (KLLTCM), the First Affiliated Hospital of Liaoning Medical University, JinZhou, \\ Liaoning; ${ }^{3}$ RSKT Biopharma Inc., Jinzhou 121000, Liaoning; ${ }^{4}$ Department of SICU, Affiliated Zhongshan Hospital of Dalian \\ University, Dalian, Liaoning; ${ }^{5}$ Department of Biotechnology, Dalian Institute of Chemical Physics, Chinese Academy \\ of Sciences, Dalian, Liaoning; 'Department of Translational Medicine Center, Clinical Laboratory, Dalian Sixth People's \\ Hospital, Dalian, Liaoning, China
}

\begin{abstract}
Background: Acute cerebral infarction $(\mathrm{ACl})$ and intracerebral hemorrhage $(\mathrm{ICH})$ are potentially lethal cerebrovascular diseases that seriously impact public health. $\mathrm{ACl}$ and $\mathrm{ICH}$ share several common clinical manifestations but have totally divergent therapeutic strategies. A poor diagnosis can affect stroke treatment. Objective: To screen for biomarkers to differentiate $\mathrm{ICH}$ from $\mathrm{ACl}$, we enrolled $129 \mathrm{ACl}$ and $128 \mathrm{ICH}$ patients and 65 healthy individuals as controls. Methods: Patients with stroke were diagnosed by computed tomography/magnetic resonance imaging, and their blood samples were obtained by fingertip puncture within $2-12 \mathrm{~h}$ after stroke initiation. We compared changes in metabolites between $\mathrm{ACl}$ and ICH using dried blood spot-based direct infusion mass spectrometry technology for differentiating $\mathrm{ICH}$ from ACl. Results: Through multivariate statistical approaches, 11 biomarkers including 3-hydroxylbutyrylcarnitine, glutarylcarnitine (C5DC), myristoylcarnitine, 3-hydroxypalmitoylcarnitine, tyrosine/citrulline (Cit), valine/phenylalanine, C5DC/3-hydroxyisovalerylcarnitine, C5DC/palmitoylcarnitine, hydroxystearoylcarnitine, ratio of sum of $\mathrm{C} 0, \mathrm{C} 2, \mathrm{C} 3, \mathrm{C} 16$, and $\mathrm{C} 18: 1$ to $\mathrm{Cit}$, and propionylcarnitine/methionine were screened. An artificial neural network model was constructed based on these parameters. A training set was evaluated by cross-validation method. The accuracy of this model was checked by an external test set showing a sensitivity of 0.8400 (95\% confidence interval [CI], 0.7394-0.9406) and specificity of 0.7692 ( $95 \% \mathrm{Cl}, 0.6536-0.8848)$. Conclusion: This study confirmed that metabolomic analysis is a promising tool for rapid and timely stroke differentiation and prediction based on differential metabolites.
\end{abstract}

Key words: Acute cerebral infarction. Artificial neural network. Intracerebral hemorrhage. Mass spectrometry. Metabolomics.

\author{
Corresponding author: \\ *Xuxin Zhang \\ Department of Neurosurgery \\ Affiliated Zhongshan Hospital of Dalian University \\ Dalian, Liaoning, China \\ E-mail: 13842623672@163.com \\ *Dongfeng Deng \\ Department of Neurosurgery \\ Affiliated Zhongshan Hospital of Dalian University \\ Dalian, Liaoning, China \\ E-mail: dongfengdeng12@163.com
}

Received for publication: 26-07-2017

Approved for publication: 06-10-2017

doi: $10.24875 / R I C .17002348$ 


\section{INTRODUCTION}

Stroke is a cerebrovascular disease that has become the major leading cause of death in adults worldwide $^{1,2}$. While stroke morbidity decreased in developed countries due to interventions during the hyperacute stage of stroke, disease morbidity has continued to increase in developing nations ${ }^{3}$. Stroke has become a heavy burden for the patient's family.

Basically, stroke can be categorized into ischemic and hemorrhagic stroke. Acute cerebral infarction (ACI) is an ischemic stroke with a high incidence, mortality, and recurrence rate ${ }^{4}$ and constitutes about $70 \%$ of all types of stroke ${ }^{5}$. Hemorrhagic stroke is represented by intracerebral hemorrhage $(\mathrm{ICH})$, which is an exceptionally devastating form of stroke that always induces severe disability and mortality ${ }^{6}$. In nearly all cases of both subtypes of stroke, there is a sudden onset without a specific aura, and the two diseases share several common clinical manifestations, such as hemiplegia, aphasia, and other neurological deficits, whereas their therapies are totally divergent. Even the treatment itself can transform ischemic stroke into hemorrhagic stroke ${ }^{7,8}$. Hence, it is crucial to distinguish $\mathrm{ICH}$ from $\mathrm{ACl}$ due to substantial differences in pathogenesis, treatment, and prognosis. Diagnosis of the two-stroke subtypes is not straightforward and is still limited. Although image-based strategies including computed tomography (CT) and magnetic resonance imaging (MRI) can be used to diagnose stroke, and achieve a relatively accurate diagnosis, they are costly, time-consuming and-dependent, complicated, not universally available and are restricted for the diagnosis of certain subtypes of stroke. Thus, extensive research has been focused on the study of blood biomarkers for the diagnosis and prognosis of stroke ${ }^{9,10}$.

Many small-molecule metabolites are changed by specific physiological or pathological status. The newly emerging metabolomic technologies can quantitatively describe the low molecular weight metabolites, examine the changed metabolite patterns from a whole set of metabolites in a specific system (body fluids or tissue) for the early diagnosis and detection of many diseases ${ }^{11}$, and provide new insights into the regulation of metabolic homeostasis in healthy and ill individuals. Metabolomic technologies have been widely used in biomarker discovery, disease stratification, and drug toxicity evaluation. Mass spectrometry
(MS) and nuclear magnetic resonance (NMR) spectroscopy are the prevailing analytical techniques applied in metabolomics and have been used in stroke research. NMR-based metabolic profiles of plasma and urine have been investigated in stroke patients showing increased levels of lactate, pyruvate, glycolate, and formate, and decreased levels of glutamine and methanol in plasma; in addition, decreased levels of citrate, hippurate, and glycine were found in the urine of these stroke patients ${ }^{10}$. Chromatography-MS, as one of the prevailing metabolomics detecting technologies, has been applied in the diagnosis of cerebral infarction, and a series of endogenous metabolites in one-carbon cycle, such as folic acid, cysteine, S-adenosyl homocysteine, and oxidized glutathione, have been identified as potential biomarkers of cerebral infarction ${ }^{12}$. The metabolomic experiments using MS platforms have become an integral part of clinical and systems biology research. To satisfy the requirement of rapid stroke diagnosis in the clinical setting, direct infusion MS technology was used in this study. The dried blood spot (DBS) sample technique has been applied in various fields, such as targeted blood metabolite analysis and newborn screening ${ }^{13,14}$. The profilings of amino acid and carnitine/acylcarnitine in DBS samples have been devoted to disease research ${ }^{15}$. In this study, the DBS-based metabolomic study was performed by direct infusion MS analysis in samples of stroke patients and healthy individuals, aimed at rapidly differentiating $\mathrm{ICH}$ from $\mathrm{ACl}$. Samples were tested for the different metabolites in $\mathrm{ACl}$ and $\mathrm{ICH}$ patients compared with healthy individuals based on the differential profiling of amino acid and carnitine/acylcarnitine.

\section{MATERIALS AND METHODS}

\section{Subjects and samples}

DBS samples from 129 patients diagnosed with $\mathrm{ACl}, 128$ with $\mathrm{ICH}$, and 65 healthy individuals were collected at the Affiliated Zhongshan Hospital of Dalian University. Patients with stroke were diagnosed by CT/MR; individuals with infection, tumor, or other serious vascular diseases were excluded. Healthy individuals had no clinically manifest diseases; cardiovascular and cerebrovascular diseases and other organic illnesses were ruled out from the healthy control group. Among the patients with $\mathrm{ICH}$, there were 76 cases with basal ganglia hemorrhage, 31 with thalamic hemorrhage, and 21 with cerebral lobar hemorrhage. The average ages of $\mathrm{ACl}$ and $\mathrm{ICH}$ 
patients and healthy individuals were 58.14 (28-76), 58.04 (33-75), and 55.68 (39-82) years, respectively. There were no age ( $p=0.06$, Kruskal-Wallis rank sum test) or gender ( $p=0.89$, Fisher's exact test) differences between the three groups. This study was conducted following the guidelines of the Ethics Committee of the Affiliated Zhongshan Hospital of Dalian University, and all the participants or their proxies signed informed consent forms. DBS samples were obtained by fingertip puncture within 2-12 h after stroke initiation; in healthy individuals, DBS samples were taken on enrollment. Eighty percent of the metabolomic data for $\mathrm{ACl}$ and $\mathrm{ICH}$ patients was randomly selected as a training set to establish a diagnosis model. There were 103 cases with $\mathrm{ACl}$ in the training set. The $\mathrm{ICH}$ in the training set was composed of $80 \%$ basal ganglia hemorrhage (61 patients), thalamic hemorrhage (25 patients), and cerebral lobar hemorrhage (17 patients) samples. The remaining $20 \%$ of samples from $\mathrm{ACl}$ and $\mathrm{ICH}$ were used to verify the applicability of the established model.

\section{Laboratory procedures}

\section{Chemicals}

High-performance liquid chromatography grade acetonitrile and high-purity water were acquired from Thermo Fisher (Waltham, MA). 1-Butanol and acetyl chloride were purchased from Sigma-Aldrich (St. Louis, MO). The isotope-labeled internal standards for 12 amino acids (catalog number: NSK-A) and 8 acylcarnitines (catalog number: NSK-B) for the purpose of absolute quantification were acquired from Cambridge Isotope Laboratories (Tewksbury, MA). These isotope standards were mixed into $2 \mathrm{~mL}$ pure methanol and stored at $4^{\circ} \mathrm{C}$. Metabolite extraction was performed through 100 -fold dilution to obtain a working solution. Amino acid and carnitine quality control (QC) standards were obtained from Chromsystems (Grafelfing, Germany). The QC samples were inserted into the real samples to ensure analysis stability.

\section{Sample preparation}

A 3-mm diameter disc was punched from DBS paper. Discs were placed into Millipore MultiScreen HV 96-well plates (Millipore, Billerica, MA, USA) to extract metabolites. The detailed sample preparation processes including QC and real sample preparations were referred to in a previous report ${ }^{16}$.

\section{Metabolomic analysis}

Direct-injection MS analysis was performed using the AB SCIEX 4000 QTrap system (Framingham, MA) with electrospray ionization source. Analytes were scanned under positive mode, and scan parameters were set as previously reported ${ }^{16}$. The injected sample was $20 \mu \mathrm{L}$. Eighty percent acetonitrile aqueous solution was used as the mobile phase. The initial flow rate was set to $0.2 \mathrm{~mL} /$ $\mathrm{min}$ and then reduced to $0.01 \mathrm{~mL} / \mathrm{min}$ within $0.08 \mathrm{~min}$. This flow rate was kept stable during $1.5 \mathrm{~min}$, subsequently increased to $0.2 \mathrm{~mL} / \mathrm{min}$ within $0.01 \mathrm{~min}$, and remained constant for $0.5 \mathrm{~min}$. The voltage for ion spray was $4.5 \mathrm{kV}$. The curtain gas pressure was 20 psi. The pressure for ion source gas 1 and gas 2 was set to 35 psi. The temperature of the auxiliary gas was set to $350^{\circ} \mathrm{C}$.

\section{Statistical analysis}

The system's control and data collection were performed using Analyst v1.6.0 software (AB Sciex). Data preprocessing was executed with ChemoView 2.0.2 (AB Sciex). Significant analysis of microarrays (SAM) was employed for screening differential metabolites. Shapiro-Wilk test was used to check the normality of variables for stroke patients and healthy individuals. ANOVA test was performed to evaluate the differentiation across various $\mathrm{ICH}$ types. The differences between stroke $(\mathrm{ACl}$ and $\mathrm{ICH}$ ) and healthy groups and between $\mathrm{ACl}$ and $\mathrm{ICH}$ were assessed by $t$-test. For the non-parametric variables, Mann-Whitney test and Kruskal-Wallis rank sum test were used for the difference evaluation. False discovery rate (FDR) was used for adjusting $p$ values for multiple hypothesis testing based on Benjamini-Hochberg correction method ${ }^{17}$. The step-wise regression analysis was used for further screening of parameters.

\section{Artificial neural network (ANN) system}

The ANN system was used to build the stroke diagnostic model. The feedforward ANN model was established by AMORE package of $\mathrm{R}$ software to distinguish $\mathrm{ICH}$ from $\mathrm{ACl}$. The ANN was performed with 11 units in the input layer, and the unit in the hidden layer was set to 10 . This 
algorithm was executed to build a model based on a training set data, and the model was evaluated by a leave-oneout cross-validation (LOO-CV) method ${ }^{18}$. Four indices were utilized to evaluate the prediction model, including sensitivity, specificity, and accuracy. The accuracy of ANN classifier was further validated via the testing set. Statistical analysis was performed with R v3.3.1 software.

\section{RESULTS}

\section{Metabolomic differences between the stroke groups and healthy individuals}

$\mathrm{ACl}$ and $\mathrm{ICH}$ were in separate compared to the healthy group to detect differentially changed metabolites between $\mathrm{ACl}$ and the healthy group, and $\mathrm{ICH}$ and the healthy group, through the SAM method; SAM results are shown in figures $\mathrm{S} 1$ and $\mathrm{S} 2$, respectively. We performed further screening of these significantly changed metabolites through further calculations and correction of the $\mathrm{p}$ values between stroke ( $\mathrm{ACl}$ and $\mathrm{ICH}$ groups) and healthy individuals. We found that 44 parameters showed different levels between $\mathrm{ACl}$ and the healthy group (Table S1), where 13 metabolites had increased levels in the $\mathrm{ACl}$ group compared to the control group. With respect to $\mathrm{ICH}$ compared to the healthy group, we found different levels in 45 parameters (Table S2), with levels of 13 metabolites increased in the ICH group compared to the control group. These metabolites screened out by FDR were used as inputs to the forward-backward step-wise regression analysis for further feature

Figure 1. Eleven parameters were significantly different between acute cerebral infarction and healthy groups (control). The mean and standard error of the mean were marked with a black line.
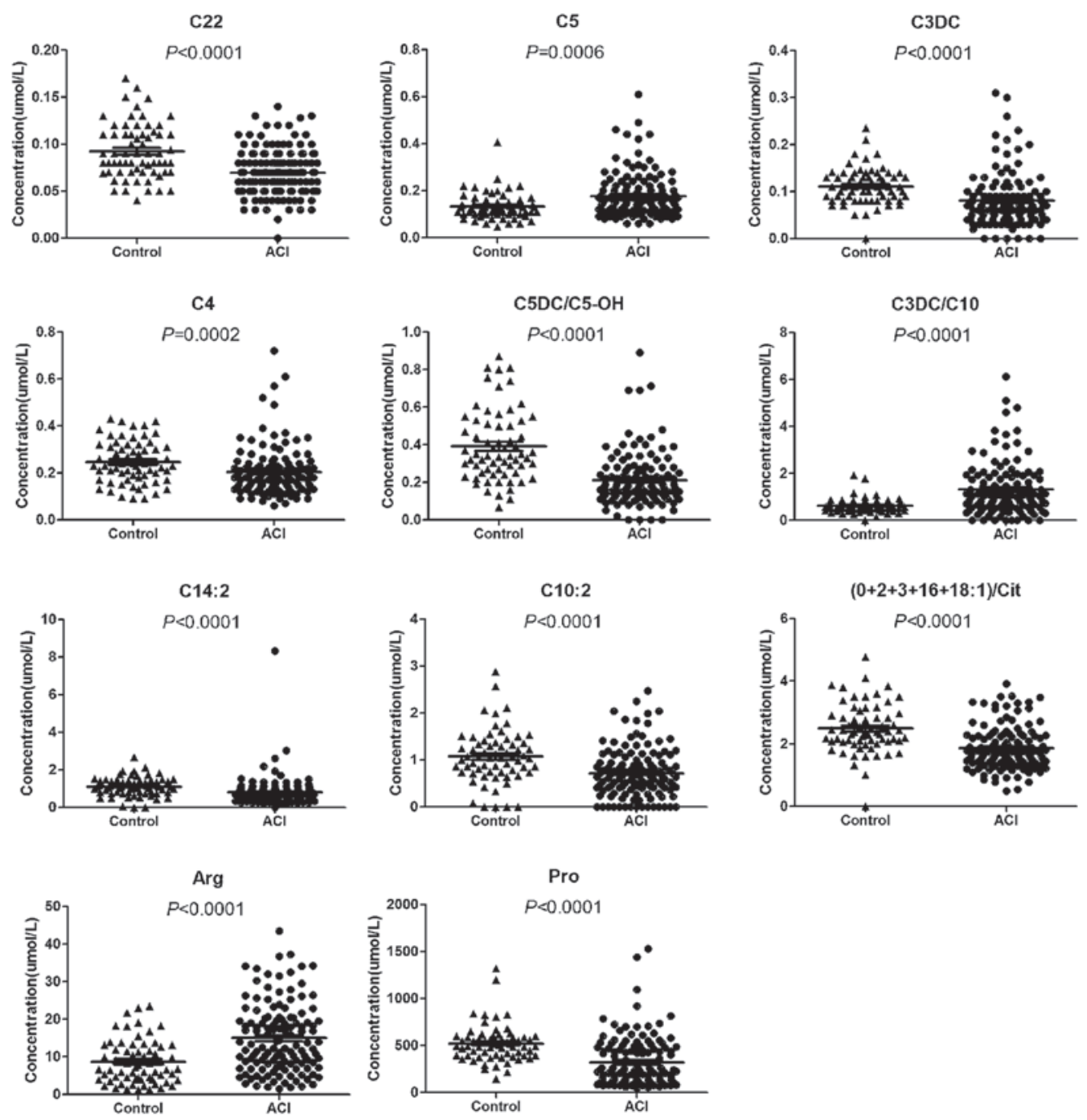
selection through $\mathrm{R}$ package MASS to differentiate $\mathrm{ACl}$ and ICH from the healthy group. Ultimately, 11 parameters were significantly different between $\mathrm{ACl}$ and the healthy group, including behenic carnitine (C22); isovalerylcarnitine (C5); C3DC, butyrylcarnitine; glutarylcarnitine (C5DC)/3-hydroxyisovalerylcarnitine (C5-OH); C3DC/decanoylcarnitine (C10); C14:2; $\mathrm{C} 10: 2$; ratio of sum of $\mathrm{C} 0, \mathrm{C} 2, \mathrm{C} 3, \mathrm{C} 16$, and $\mathrm{C} 18: 1$ to citrulline $[(0+2+3+16+18: 1) / C i t]$; arginine (Arg); and proline (Pro), where the levels of $\mathrm{C} 5, \mathrm{C} 3 \mathrm{DC} / \mathrm{C} 10$, and Arg were increased in $\mathrm{ACl}$ patients compared to the healthy group (Fig. 1). Furthermore, when comparing the $\mathrm{ICH}$ group to the control group, 12 parameters were significantly different, including 3-hydroxypalmitoylcarnitine $(\mathrm{C} 16-\mathrm{OH}) /$ palmitoylcarnitine $(\mathrm{C} 16)$, 3-hydroxypalmitoleylcarnitine $\quad(\mathrm{C} 16: 1-\mathrm{OH}), \quad \mathrm{C} 10$, $\mathrm{C} 5 /$ propionylcarnitine (C3), lauroylcarnitine (C12), octadecanoylcarnitine (C18), oleylcarnitine (C18:1), succinyl-/methylmalonylcarnitine, valine (Val)/phenylalanine (Phe), C16, Arg, and threonine (Thr); among them, the levels of 3 parameters $(\mathrm{C} 16-\mathrm{OH} / \mathrm{C} 16, \mathrm{C} 5 /$ $\mathrm{C} 3$, and $\mathrm{Arg}$ ) were increased in the $\mathrm{ICH}$ group compared to the healthy group (Fig. 2). These results show that the affected metabolites were almost different in $\mathrm{ACl}$ and $\mathrm{ICH}$, especially for the carnitine-related metabolites, which suggest that the pathological progression of $\mathrm{ACl}$ and $\mathrm{ICH}$ may be different.

\section{Metabolomic analysis for $\mathrm{ACl}$ and $\mathrm{ICH}$ groups}

To further explore the discrepancy between $\mathrm{ACl}$ and $\mathrm{ICH}$, we also performed a metabolomic analysis for the

Figure 2. Twelve parameters were significantly different between intracerebral hemorrhage and healthy groups (control).
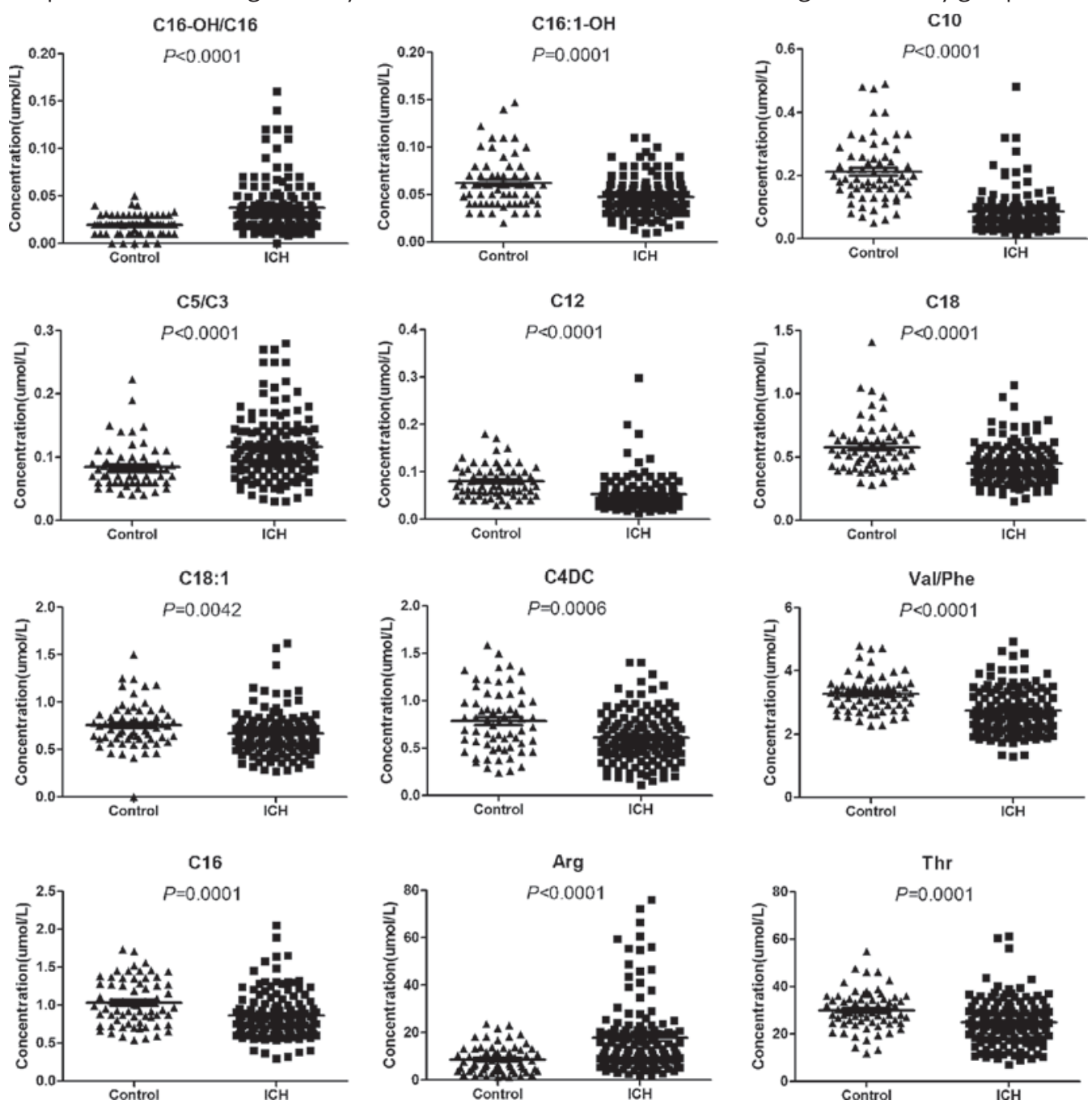
two groups, finding that this method could distinguish $\mathrm{ICH}$ from the $\mathrm{ACl}$ group. First, SAM screening (Fig. 3) was integrated with the $p$ value calculation and FDR correction to differentiate $\mathrm{ICH}$ from $\mathrm{ACl}$. Ultimately, 37 significantly differentiated metabolites were screened out (Table 1), where 15 of these metabolites were increased in the $\mathrm{ICH}$ group compared to the $\mathrm{ACl}$ group. Second, these selected metabolites were subjected to step-wise regression for further screening of differentiated metabolites. Finally, 11 significantly differentiated metabolites were selected to build a prediction model, including 3-hydroxylbutyrylcarnitine $(\mathrm{C} 4-\mathrm{OH}), \mathrm{C} 5 \mathrm{DC}$, myristoylcarnitine (C14), $\mathrm{C} 16-\mathrm{OH}$, tyrosine (Tyr)/Cit, $\mathrm{Val} / \mathrm{Phe}, \mathrm{C} 5 \mathrm{DC} / \mathrm{C} 5-\mathrm{OH}, \mathrm{C} 5 \mathrm{DC} / \mathrm{C} 16$, hydroxystearoylcarnitine $(\mathrm{C} 18-\mathrm{OH}),(0+2+3+16+18: 1) / \mathrm{Cit}$, and $\mathrm{C} 3 /$ methionine (Met) (Fig. 4). The levels of $\mathrm{C} 4-\mathrm{OH}, \mathrm{C} 5 \mathrm{DC}$, $\mathrm{C} 5 \mathrm{DC} / \mathrm{C} 5-\mathrm{OH}, \mathrm{C} 5 \mathrm{DC} / \mathrm{C} 16,(0+2+3+16+18: 1) / \mathrm{Cit}$, and $\mathrm{C} 3 /$ Met were elevated in the $\mathrm{ICH}$ group compared to the $\mathrm{ACl}$ group. By contrast, the levels of $\mathrm{C} 14, \mathrm{C} 16-\mathrm{OH}, \mathrm{Tyr} / \mathrm{Cit}, \mathrm{Val} / \mathrm{Phe}$, and $\mathrm{C} 18-\mathrm{OH}$ were decreased in the $\mathrm{ICH}$ group.

\section{Sample classification using ANN algorithm}

The 11 significantly differentiated metabolites were selected as predictors through step-wise regression for differentiating $\mathrm{ACl}$ from $\mathrm{ICH}$. Subsequently, the ANN model was built based on these differentiated metabolites, and the accuracy of this model was checked. For the training, LOO-CV of training, and testing sets, $26(13 \mathrm{ACl}$ and $13 \mathrm{ICH}), 44(21 \mathrm{ACl}$ and $23 \mathrm{ICH})$, and $12(8 \mathrm{ACl}$ and $4 \mathrm{ICH})$ cases, respectively, were incorrectly classified by the ANN algorithm. The accuracy of this classifier was higher than 0.7 (Table 2), and for distinguishing $\mathrm{ICH}$ from $\mathrm{ACl}$, the accuracies were 0.8738 , 0.7864 , and 0.7647 in the training, LOO-CV of training, and testing sets, respectively. In addition, the accuracies of differentiating $\mathrm{ICH}$ with different sites of bleeding from $\mathrm{ACl}$ were all higher than 0.8594 in the training set, indicating the acceptability of the ANN model. The training set was further tested by the LOO-CV method, with an accuracy of higher than 0.7734 , and the external validation for the testing set was shown with an accuracy of higher than 0.7073 . For the training, LOO-CV of training, and testing sets, the receiver operating characteristic (ROC) curve analysis was also performed to examine the diagnosis ability of the ANN model (Fig. 5a-c). In additional, the differentiation of basal ganglia hemorrhage, thalamic hemorrhage, and cerebral lobar hemorrhage with $\mathrm{ACl}$ was also examined by ROC curve analysis (Fig. 5a-c). The results of the sensitivity and specificity in the training set were higher than 0.8 for distinguishing $\mathrm{ICH}$, basal ganglia hemorrhage, thalamic hemorrhage, and cerebral lobar hemorrhage from $\mathrm{ACl}$ (Table 2), which implied the acceptability of the ANN model for clinical application. The training set was further tested by the LOO-CV method, and the sensitivity and specificity values were all higher than 0.7600 . The external validation was implemented for the testing set, and the sensitivity and specificity values were all higher than 0.7333 .

\section{DISCUSSION}

Stroke is a major public health problem with a poor prognosis. The changes in metabolite levels in biofluids are regarded to be in accord with the metabolic alterations in the brain ${ }^{19}$. Using DBS-based metabolomic assay coupled with multivariate statistics analysis, we found that several metabolites including amino acids, acylcarnitines, or their ratios were altered among $\mathrm{ACl}$, $\mathrm{ICH}$, and healthy individuals. These changed metabolites not only reflected the systemic deregulation during the course of $\mathrm{ACl}$ or $\mathrm{ICH}$ but also represented the metabolic status in the brain ${ }^{20,21}$. In addition, these changed metabolites could also be used in the prediction, diagnosis, or prognosis of stroke.

Figure 3. Significant analysis of microarrays for metabolomic data between intracerebral hemorrhage $(\mathrm{ICH})$ and acute cerebral infarction $(\mathrm{ACl})$ with false discovery rate set to zero. The dots above truncation mean the significantly increased metabolites in $\mathrm{ICH}$ group compared to $\mathrm{ACl}$ group. The dots below truncation mean the significantly decreased metabolites in $\mathrm{ICH}$ group compared to $\mathrm{ACl}$ group.

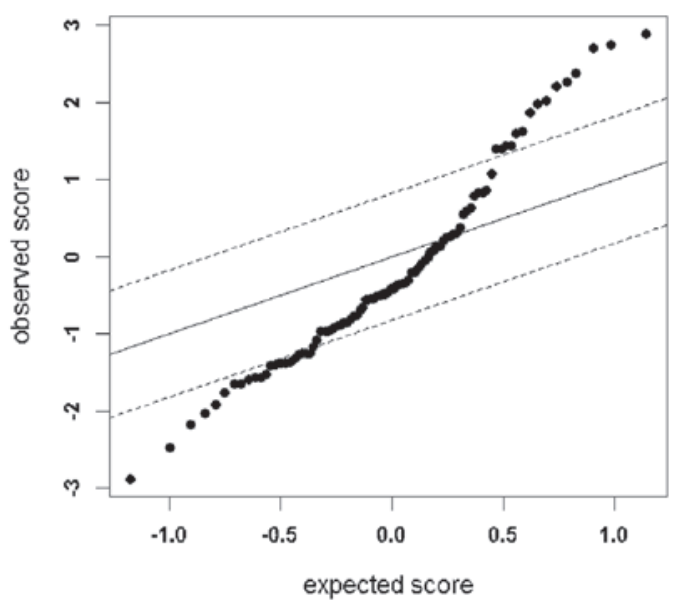


Table 1. Differential parameters between $\mathrm{ACl}$ and $\mathrm{ICH}$ groups after SAM screening and FDR correction.

\begin{tabular}{|c|c|c|c|c|c|c|}
\hline No. & Parameters & $\mathrm{ACl}($ mean $\pm \mathrm{SD})$ & $\mathrm{ICH}($ mean $\pm \mathrm{SD})$ & Status & $p$ value & FDR \\
\hline 1 & C5DC/C16 & $0.0542 \pm 0.0317$ & $0.0931 \pm 0.0619$ & $\uparrow$ & 0.0000 & 0.0000 \\
\hline 2 & Phe/Tyr & $0.7554 \pm 0.4044$ & $1.0604 \pm 0.3639$ & $\uparrow$ & 0.0000 & 0.0000 \\
\hline 3 & $\mathrm{C} 5 \mathrm{DC} / \mathrm{C} 5-\mathrm{OH}$ & $0.2018 \pm 0.1196$ & $0.3282 \pm 0.1937$ & $\uparrow$ & 0.0000 & 0.0000 \\
\hline 4 & $\mathrm{C} 5 \mathrm{DC} / \mathrm{C} 8$ & $0.8563 \pm 0.4976$ & $1.4292 \pm 1.0531$ & $\uparrow$ & 0.0000 & 0.0000 \\
\hline 5 & $\mathrm{C} 3 / \mathrm{C} 16$ & $1.3953 \pm 0.6330$ & $1.9119 \pm 0.9395$ & $\uparrow$ & 0.0000 & 0.0001 \\
\hline 6 & C5DC & $0.0509 \pm 0.0297$ & $0.0732 \pm 0.0425$ & $\uparrow$ & 0.0000 & 0.0001 \\
\hline 7 & $\mathrm{C} 2 / \mathrm{CO}$ & $0.3181 \pm 0.1285$ & $0.4247 \pm 0.2214$ & $\uparrow$ & 0.0001 & 0.0004 \\
\hline 8 & C3DC & $0.0831 \pm 0.0561$ & $0.1135 \pm 0.1100$ & $\uparrow$ & 0.0001 & 0.0006 \\
\hline 9 & C3/Met & $0.0714 \pm 0.0348$ & $0.0956 \pm 0.0507$ & $\uparrow$ & 0.0002 & 0.0012 \\
\hline 10 & $C 26 / C 20$ & $0.7480 \pm 0.2994$ & $1.0323 \pm 0.6491$ & $\uparrow$ & 0.0012 & 0.0061 \\
\hline 11 & $\mathrm{C} 2$ & $10.0578 \pm 4.0909$ & $12.5465 \pm 5.7029$ & $\uparrow$ & 0.0013 & 0.0065 \\
\hline 12 & $\mathrm{C} 3 / \mathrm{CO}$ & $0.0413 \pm 0.0187$ & $0.0529 \pm 0.0326$ & $\uparrow$ & 0.0037 & 0.0145 \\
\hline 13 & $\mathrm{C} 4-\mathrm{OH}$ & $0.0810 \pm 0.0524$ & $0.1174 \pm 0.1046$ & $\uparrow$ & 0.0040 & 0.0148 \\
\hline 14 & $(0+2+3+16+18: 1) /$ Cit & $1.9198 \pm 0.7236$ & $2.2929 \pm 0.9723$ & $\uparrow$ & 0.0049 & 0.0166 \\
\hline 15 & Arg/Orn & $0.4853 \pm 0.4348$ & $0.6374 \pm 0.4459$ & $\uparrow$ & 0.0051 & 0.0166 \\
\hline 16 & Val/Phe & $3.3963 \pm 0.6886$ & $2.7791 \pm 0.7313$ & $\downarrow$ & 0.0000 & 0.0000 \\
\hline 17 & $\mathrm{C} 18: 1-\mathrm{OH}$ & $0.0365 \pm 0.0170$ & $0.0268 \pm 0.1516$ & $\downarrow$ & 0.0000 & 0.0000 \\
\hline 18 & Tyr/Cit & $1.8249 \pm 0.7262$ & $1.4139 \pm 0.7044$ & $\downarrow$ & 0.0000 & 0.0001 \\
\hline 19 & Met/Phe & $0.4410 \pm 0.1078$ & $0.3738 \pm 0.1169$ & $\downarrow$ & 0.0000 & 0.0004 \\
\hline 20 & $\mathrm{C} 5 / \mathrm{C} 2$ & $0.0204 \pm 0.0130$ & $0.0148 \pm 0.0086$ & $\downarrow$ & 0.0001 & 0.0006 \\
\hline 21 & $\mathrm{C} 18-\mathrm{OH}$ & $0.0301 \pm 0.0145$ & $0.0238 \pm 0.0140$ & $\downarrow$ & 0.0003 & 0.0021 \\
\hline 22 & Val & $153.4299 \pm 41.6956$ & $133.8167 \pm 42.1753$ & $\downarrow$ & 0.0010 & 0.0055 \\
\hline 23 & Thr & $30.4741 \pm 11.7428$ & $25.2498 \pm 10.2961$ & $\downarrow$ & 0.0010 & 0.0055 \\
\hline 24 & Orn & $68.2662 \pm 55.7599$ & $48.6449 \pm 53.4322$ & $\downarrow$ & 0.0015 & 0.0071 \\
\hline 25 & Lys & $168.7855 \pm 80.0643$ & $136.9643 \pm 87.2784$ & $\downarrow$ & 0.0018 & 0.0078 \\
\hline 26 & Leu & $138.2196 \pm 43.4851$ & $120.8855 \pm 52.8319$ & $\downarrow$ & 0.0018 & 0.0078 \\
\hline 27 & $\mathrm{C} 5 / \mathrm{C} 3$ & $0.1527 \pm 0.0918$ & $0.1147 \pm 0.0521$ & $\downarrow$ & 0.0024 & 0.0098 \\
\hline 28 & $\mathrm{C} 16$ & $0.9833 \pm 0.3325$ & $0.8594 \pm 0.3088$ & $\downarrow$ & 0.0048 & 0.0166 \\
\hline 29 & $\mathrm{C} 20$ & $0.0503 \pm 0.0187$ & $0.0433 \pm 0.0225$ & $\downarrow$ & 0.0052 & 0.0166 \\
\hline 30 & C14 & $0.0832 \pm 0.0879$ & $0.0642 \pm 0.0242$ & $\downarrow$ & 0.0055 & 0.0166 \\
\hline 31 & Cit/Arg & $2.4253 \pm 1.7677$ & $1.9476 \pm 1.7663$ & $\downarrow$ & 0.0059 & 0.0172 \\
\hline 32 & C10:1 & $0.13912 \pm 0.1149$ & $0.1019 \pm 0.0657$ & $\downarrow$ & 0.0064 & 0.0181 \\
\hline 33 & $\mathrm{C} 8 / \mathrm{C} 2$ & $0.0081 \pm 0.0065$ & $0.0059 \pm 0.0053$ & $\downarrow$ & 0.0054 & 0.0166 \\
\hline 34 & C18:2 & $1.9413 \pm 0.6619$ & $1.7459 \pm 0.7187$ & $\downarrow$ & 0.0111 & 0.0303 \\
\hline 35 & $\mathrm{C} 16-\mathrm{OH}$ & $0.0431 \pm 0.0310$ & $0.0336 \pm 0.0251$ & $\downarrow$ & 0.0121 & 0.0313 \\
\hline 36 & Cit & $25.9877 \pm 10.407$ & $23.4265 \pm 12.0841$ & $\downarrow$ & 0.0125 & 0.0313 \\
\hline 37 & Met & $20.3045 \pm 6.5841$ & $18.0685 \pm 7.0254$ & $\downarrow$ & 0.0125 & 0.0313 \\
\hline
\end{tabular}

$\mathrm{ACl}$ : acute cerebral infarction; ICH: intracerebral hemorrhage; SAM: significant analysis of microarrays; FDR: false discovery rate; SD: standard deviation; Tyr: tyrosine; Cit: citrulline; Val: valine; Phe: phenylalanine; Met: methionine; Arg: arginine; Thr: threonine; $\mathrm{CO}$ : free carnitine; $\mathrm{C} 2$ : acetylcarnitine; $\mathrm{C}$ : propionylcarnitine; $\mathrm{C} 4-\mathrm{OH}$ : 3-hydroxylbutyrylcarnitine; $\mathrm{C}$ : cisovalerylcarnitine; C5DC: glutarylcarnitine; $\mathrm{C5}-\mathrm{OH}$ : 3-hydroxyisovalerylcarnitine; C14: myristoylcarnitine; C16: palmitoylcarnitine; $\mathrm{C} 18-\mathrm{OH}$ : hydroxystearoylcarnitine; $(0+2+3+16+18: 1) /$ Cit: ratio of sum of $\mathrm{C} 0, \mathrm{C} 2, \mathrm{C} 3, \mathrm{C} 16$, and $\mathrm{C} 18: 1$ to $\mathrm{Cit}$

Amino acids as the substrates of protein synthesis are essential to human function. Metabolomic investigations have shown abnormalities of amino acid metabolism in stroke development ${ }^{10,22}$. The supervised method we describe here was performed to study the differences in changed metabolites between $\mathrm{ACl}$ and $\mathrm{ICH}$ patients and healthy individuals. The results revealed that the level of Arg was elevated in both $\mathrm{ACl}$ and $\mathrm{ICH}$ patients. The level of this amino acid has been found increased in acute ischemic stroke patients and showed a positive correlation with the risk factors for acute ischemic stroke ${ }^{23}$. Arginase and nitric oxide synthase (NOS) competed for the common substrate
Arg. The significant effects of arginase on circulation implied the potential role of this enzyme to regulate vascular function ${ }^{24}$. It is worth noting that the increased arginase leads to arginase/NOS imbalance and reduces NO production, thus generating endothelial dysfunction, and may also influence the utilization of the substrate Arg ${ }^{25,26}$. In addition, we found that prometabolism was abnormal in $\mathrm{ACl}$ patients, which was consistent with the previous reports demonstrating that Pro was markedly reduced in acute ischemic stroke patients ${ }^{27,28}$, and showed a connection to blood stasis in coronary heart disease ${ }^{29}$. Phenylalanine hydroxylase $(\mathrm{PAH})$ could catalyze the hydroxylation 
Figure 4. Levels of 11 metabolites in the artificial neural network (ANN) model. The 11 metabolites were used to build ANN model for distinguishing intracerebral hemorrhage from acute cerebral infarction.
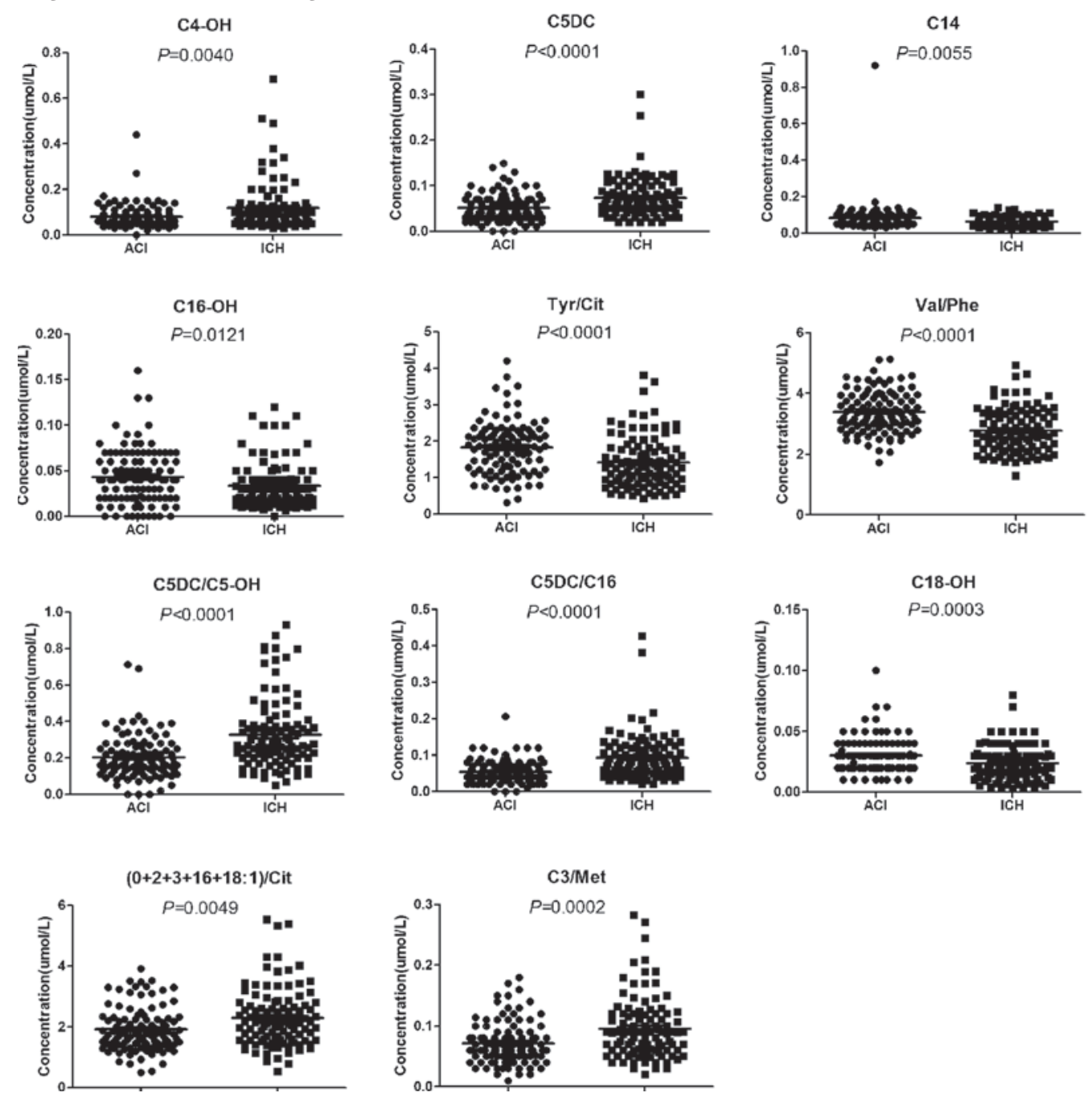

of aromatic side-chain of Phe and produce Tyr. The stroke-associated oxidative stress may decrease PAH after stroke occurred, which could induce the accumulation of Phe, leading to the decrease of the ratio $\mathrm{Val} /$ Phe in $\mathrm{ICH}$ patients. Val, as one of the branchedchain amino acids (BCAA), can influence brain function through energy production and neurotransmitter synthesis $^{30}$. Furthermore, BCAAs were found to be decreased in several diseases, such as sepsis, brain injury, and heart diseases ${ }^{22,31}$. The decreased value of $\mathrm{Val} /$ Phe radio was probably not only influenced by the changed level of Phe, but also by the decreased level of Val in $\mathrm{ICH}$ patients. Thr was significantly decreased in $\mathrm{ICH}$ patients, and this result was consistent with a previous report ${ }^{28}$. Thr can be converted to pyruvate by glycolysis for energy supply. The reduction of Thr levels may impact its utilization and further affect brain function ${ }^{28}$.

We found that metabolites such as carnitine and some acetylcarnitine (C2) were markedly altered in stroke patients. During lipid catabolism, carnitine transports fatty acids into the mitochondria for converting fat into energy. C2 is synthesized by transferring an acetyl group to carnitine, and this reaction facilitates its crossing of the blood-brain barrier. The levels of two longchain acylcarnitines (C14:2 and C22) were decreased in $\mathrm{ACl}$ patients, while the levels of more long-chain acylcarnitines declined in $\mathrm{ICH}$ patients, including $\mathrm{C} 12$, $\mathrm{C} 16, \mathrm{C} 18, \mathrm{C} 18: 1$, and $\mathrm{C} 16: 1-\mathrm{OH}$. It has been found that the decreased carnitine-palmitoyltransferase1 (CPT1) is accompanied by the decrease of acylcarnitines, 
Figure 5. Receiver operating characteristic (ROC) curve based on artificial neural network model of training set (a), Leave-one-out cross-validation of training set (b), and testing set (c). The cases of intracerebral hemorrhage used for building ROC curve included basal ganglia hemorrhage, thalamic hemorrhage, and cerebral lobar hemorrhage patients.
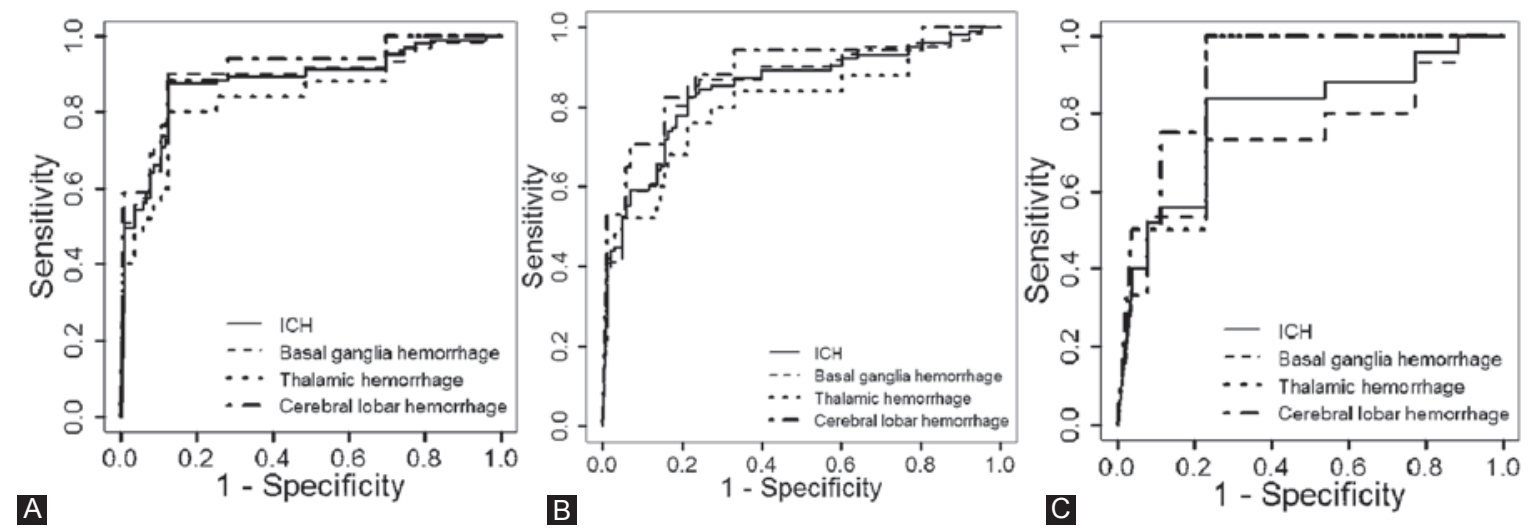

Table 2. Sensitivity, specificity, and accuracy values for distinguishing $\mathrm{ICH}$, and $\mathrm{ICH}$ with different sites of bleeding from $\mathrm{ACl}$ from the training set, LOO-CV of training set, and testing set.

\begin{tabular}{|c|c|c|c|}
\hline Model & Sensitivity $(95 \% \mathrm{Cl})$ & Specificity $(95 \% \mathrm{Cl})$ & Accuracy \\
\hline \multicolumn{4}{|l|}{ Training } \\
\hline $\mathrm{ICH}^{\mathrm{a}}$ & $0.8738(0.8285-0.9191)$ & $0.8738(0.8285-0.9191)$ & 0.8738 \\
\hline Basal ganglia hemorrhage & $0.9016(0.8560-0.9472)$ & $0.8738(0.8230-0.9246)$ & 0.8841 \\
\hline Thalamic hemorrhage & $0.8000(0.7307-0.8693)$ & $0.8738(0.8163-0.9313)$ & 0.8594 \\
\hline Cerebral lobar hemorrhage & $0.8824(0.8248-0.9400)$ & $0.8738(0.8144-0.9332)$ & 0.8750 \\
\hline \multicolumn{4}{|l|}{ Training (LOO) } \\
\hline $\mathrm{ICH}^{\mathrm{a}}$ & $0.8252(0.7733-0.8771)$ & $0.7864(0.7304-0.8424)$ & 0.7864 \\
\hline Basal ganglia hemorrhage & $0.8525(0.7982-0.9068)$ & $0.7864(0.7237-0.8491)$ & 0.7988 \\
\hline Thalamic hemorrhage & $0.7600(0.6860-0.8340)$ & $0.7864(0.7154-0.8574)$ & 0.7734 \\
\hline Cerebral lobar hemorrhage & $0.8235(0.7553-0.8917)$ & $0.8447(0.7799-0.9095)$ & 0.8000 \\
\hline \multicolumn{4}{|l|}{ Testing } \\
\hline $\mathrm{ICH}^{\mathrm{a}}$ & $0.8400(0.7394-0.9406)$ & $0.7692(0.6536-0.8848)$ & 0.7647 \\
\hline Basal ganglia hemorrhage & $0.7333(0.5979-0.8687)$ & $0.7692(0.6402-0.8982)$ & 0.7073 \\
\hline Thalamic hemorrhage & $1.0000(0.5168-1.0000)$ & $0.7692(0.6232-0.9152)$ & 0.7500 \\
\hline Cerebral lobar hemorrhage & $1.0000(0.3958-1.0000)$ & $0.7692(0.6184-0.9200)$ & 0.7333 \\
\hline
\end{tabular}

$\mathrm{ACl}$ : acute cerebral infarction; $\mathrm{ICH}$ : intracerebral hemorrhage; LOO-CV: leave-one-out cross-validation; $\mathrm{Cl}$ : confidence interval a $\mathrm{CH}$ group was composed of the patients with basal ganglia hemorrhage, thalamic hemorrhage, and cerebral lobar hemorrhage

especially for the long-chain acylcarnitines, in the heart of cyclophilin $\mathrm{D}^{-/-}$mice ${ }^{32}$. CPT1 is located on the outer mitochondrial membrane and is responsible for the transference of the acyl group from cytosolic acyl-CoA to carnitine to generate acylcarnitine. In addition, carnitine-palmitoyltransferase2 (CPT2), on the inner mitochondrial membrane, is used to transfer the acyl group to free CoA to obtain free carnitine. Through these processes, acyl-CoA is transferred into the mitochondria to conduct $\beta$-oxidation ${ }^{32}$. Therefore, the decreased level or activity of CPT 1 can reduce $\beta$-oxidation and the levels of long-chain acylcarnitines. In this study, for ICH patients, the decreased levels of long-chain acylcarnitines may be owing to the decreased level or activity of CPT1 enzyme, and the decreased CPT1 could further reduce the oxidation of fatty acids.
It is not surprising that combinational metabolites were needed in this study to differentiate stroke types due to the complexity of the neurobiological mechanisms of stroke. Through multiple screening processes, $\mathrm{C} 4-\mathrm{OH}$, C5DC, C14, C16-OH, Tyr/Cit, Val/Phe, C5DC/C5-OH, $\mathrm{C} 5 \mathrm{DC} / \mathrm{C} 16, \mathrm{C} 18-\mathrm{OH},(0+2+3+16+18: 1) / \mathrm{Cit}$, and $\mathrm{C} 3 /$ Met were selected to be potential biomarkers to build the ANN model for distinguishing $\mathrm{ICH}$ from $\mathrm{ACl}$. This model could not only precisely predict the diagnosis in $87.38 \%$ of 206 patients in the training set, but also distinguish ICH patients with different sites of bleeding from $\mathrm{ACl}$ patients. Compared to the traditional imaging tools and biochemical analyses, these potential biomarkers selected from metabolomic detection and analysis are faster to obtain and less invasive, have higher sensitivity and specificity, and show potential to expand their application. 
In this study, we discovered novel effective biomarkers by DBS-based MS metabolomic analysis to differentiate $\mathrm{ICH}$ from $\mathrm{ACl}$, and these biomarkers could even distinguish $\mathrm{ICH}$ with different sites of bleeding from $\mathrm{ACl}$ through probing changed metabolites. The LOO-CV method was used to verify the training set, and optimization of potential biomarkers was validated by a test set. The ANN prognosis model built by selecting 11 biomarkers provided a new rapid and sensitive strategy for differentiating $\mathrm{ICH}$ from $\mathrm{ACl}$. Nevertheless, the limited sample size of this study affects the prediction accuracy. In the following study, we will recruit more participants to increase the current sample, verify the findings of this study, and confirm the value of these 11 metabolites as clinical biomarkers.

\section{ACKNOWLEDGMENTS}

This study was supported by the National Natural Science Foundation of China (81672498).

\section{Supplementary data}

Supplementary data are available at Revista de Investigación Clínica online (www.clinicalandtranslationalinvestigation.com). These data are provided by the corresponding author and published online for the benefit of the reader. The contents of supplementary data are the sole responsibility of the authors.

\section{REFERENCES}

1. Towfighi A, Saver JL. Stroke declines from third to fourth leading cause of death in the United States: Historical perspective and challenges ahead. Stroke. 2011;42:2351-5.

2. O'Donnell MJ, Xavier D, Liu L, et al. Risk factors for ischaemic and intracerebral haemorrhagic stroke in 22 countries (the INTERSTROKE study): A case-control study. Lancet. 2010; 376:112-23.

3. Hu SL, Feng H, Xi GH. Hyperbaric oxygen therapy and preconditioning for ischemic and hemorrhagic stroke. Med Gas Res. 2016;6:232-6

4. van der Worp HB, van Gijn J. Clinical practice. Acute ischemic stroke. N Engl J Med. 2007;357:572-9.

5. Luo J. New progress on treating acute cerebral infarction. Pract J Cardiac Cereb Pneumal Vasc Dis. 2010;18:825-9.

6. Keep RF, Hua Y, Xi G. Intracerebral haemorrhage: Mechanisms of injury and therapeutic targets. Lancet Neurol. 2012;11:720-31.

7. Zhang J, Yang Y, Sun H, Xing Y. Hemorrhagic transformation after cerebral infarction: Current concepts and challenges. Ann Transl Med. 2014;2:81.

8. Martinez B, Peplow PV. Blood microRNAs as potential diagnostic markers for hemorrhagic stroke. Neural Regen Res. 2017; 12:13-18.
9. Graham GD, Blamire AM, Howseman AM, et al. Proton magnetic resonance spectroscopy of cerebral lactate and other metabolites in stroke patients. Stroke. 1992;23:333-40.

10. Jung JY, Lee HS, Kang DG, et al. 1H-NMR-based metabolomics study of cerebral infarction. Stroke. 2011;42:1282-8.

11. Gowda GA, Zhang S, Gu H, et al. Metabolomics-based methods for early disease diagnostics. Expert Rev Mol Diagn. 2008;8:617-33.

12. Jiang Z, Sun J, Liang Q, et al. A metabonomic approach applied to predict patients with cerebral infarction. Talanta. 2011; 84:298-304

13. Shah NM, Hawwa AF, Millership JS, Collier PS, McElnay JC. A simple bioanalytical method for the quantification of antiepileptic drugs in dried blood spots. J Chromatogr B Analyt Technol Biomed Life Sci. 2013;923:65-73.

14. Therrell BL, Adams J. Newborn screening in North America. J Inherit Metab Dis. 2007;30:447-65.

15. Zhao D, Han L, He Z, Zhang J, Zhang Y. Identification of the plasma metabolomics as early diagnostic markers between biliary atresia and neonatal hepatitis syndrome. PLoS One. 2014;9:e85694

16. Wang Q, Sun T, Cao Y, et al. A dried blood spot mass spectrometry metabolomic approach for rapid breast cancer detection. Onco Targets Ther. 2016;9:1389-98.

17. Benjamini $Y$, Hochberg $Y$. Controlling the false discovery rate: $A$ practical and powerful approach to multiple testing. J Roy Stat Soc B. 1995;57:289-300.

18. Cawley GC, Talbot NL. Efficient leave-one-out cross-validation of kernel fisher discriminant classifiers. Pattern Recognit. 2003;36:2585-92.

19. Ivanisevic J, Siuzdak G. The role of metabolomics in brain metabolism research. J Neuroimmune Pharmacol. 2015;10:391-5.

20. Sun H, Zhang A, Yan G, et al. Metabolomic analysis of key regulatory metabolites in hepatitis $C$ virus-infected tree shrews. Mol Cell Proteomics. 2013;12:710-9.

21. ladecola C, Anrather J. Stroke research at a crossroad: Asking the brain for directions. Nat Neurosci. 2011;14:1363-8.

22. Kimberly WT, Wang Y, Pham L, Furie KL, Gerszten RE. Metabolite profiling identifies a branched chain amino acid signature in acute cardioembolic stroke. Stroke. 2013;44:1389-95.

23. Hosinian M, Qujeq D, Ahmadi Ahangar A. The relation between GABA and L-Arginine levels with some stroke risk factors in acute ischemic stroke patients. Int J Mol Cell Med. 2016; 5:100-5.

24. Nishimura H, Rosenblum WI, Nelson GH, Boynton S. Agents that modify EDRF formation alter antiplatelet properties of brain arteriolar endothelium in vivo. Am J Physiol. 1991;261: H15-21.

25. Lim HK, Lim HK, Ryoo S, et al. Mitochondrial arginase II constrains endothelial NOS-3 activity. Am J Physiol Heart Circ Physiol. 2007;293:H3317-24.

26. Steppan J, Nyhan D, Berkowitz DE. Development of novel arginase inhibitors for therapy of endothelial dysfunction. Front Immunol. 2013;4:278.

27. Wang D, Kong J, Wu J, Wang X, Lai M. GC-MS-based metabolomics identifies an amino acid signature of acute ischemic stroke. Neurosci Lett. 2017;642:7-13.

28. Liu P, Li R, Antonov AA, Wang L, Li W. Discovery of metabolite biomarkers for acute ischemic stroke progression. J Proteome Res. 2017;16:773-9.

29. Jian WX, Yuan ZK, Huang XP. Detection and analysis on plasma metabolomics in patient with coronary heart disease of Xinblood stasis syndrome pattern. Zhongguo Zhong Xi Yi Jie He Za Zhi. 2010;30:579-84.

30. Fernstrom JD. Branched-chain amino acids and brain function. J Nutr. 2005;135:1539S-46.

31. Huang $Y$, Zhou M, Sun H, Wang Y. Branched-chain amino acid metabolism in heart disease: An epiphenomenon or a real culprit? Cardiovasc Res. 2011;90:220-3.

32. Menazza S, Wong R, Nguyen T, et al. CypD(-/-) hearts have altered levels of proteins involved in Krebs cycle, branch chain amino acid degradation and pyruvate metabolism. J Mol Cell Cardiol. 2013;56:81-90. 